\title{
A Bayesian Modelling Approach to Examine the Role of Testosterone Administration on the Endowment Effect and Risk-Taking
}

\author{
Mikhail Votinov ${ }^{1,2,3 * 凶}$, Irina Knyazeva ${ }^{3,4^{*}}$, Ute Habel ${ }^{1,2}$, Kerstin Konrad $^{5}$, and Andrei A Puiu ${ }^{2 凶}$ \\ ${ }^{1}$ Institute of Neuroscience and Medicine: JARA-Institute Brain Structure Function Relationship (INM 10), Research Center Jülich, Jülich, Germany \\ ${ }^{2}$ Department of Psychiatry, Psychotherapy and Psychosomatics, Faculty of Medicine, RWTH Aachen University, Aachen, Germany \\ ${ }^{3}$ N.P. Bechtereva Institute of Human Brain, Russian Academy of Science, Saint-Petersburg, Russia \\ ${ }^{4}$ Saint-Petersburg State University, St.Petersburg, Russia \\ ${ }^{5}$ Department of Child and Adolescent Psychiatry, Psychosomatics and Psychotherapy, Faculty of Medicine, RWTH Aachen University, Aachen, Germany \\ authors contributed equally
}

\begin{abstract}
We investigated the effects of testosterone administration on aspects of decision-making within the Prospect Theory framework. Using bayesian modeling, we assessed risk-taking under framing and Endowment Effect (effect of possession). We administered $100 \mathrm{mg}$ testosterone to forty men in a doubleblind placebo-controlled fully-randomized cross-over experiment where they participated in two tasks. One was a risktaking task with binary choices under positive and negative framing with different probabilities. In a second task, participants had to bid for for two categories of items, hedonic and utilitarian. We observed a significant increase in serum testosterone concentrations after transdermal application. Compared to placebo, testosterone administration increased risk-taking under the positive framing and decreased under the negative framing. The sensitivity to gain was positive in each framing. Our model showed that decision-making is jointly influenced by testosterone and the trade-off between gains and losses. Moreover, while the endowment effect was more pronounced for hedonic than for utilitarian items, the effect was independent of testosterone. The findings provide novel information for the complex modulatory role of testosterone on risk-taking. The proposed models of effects of individual differences in testosterone on risk-taking could be used as predictive models.
\end{abstract}

testosterone | sex hormones | bayesian analysis and modelling | decision making | risk-taking | prospect theory | endowment effect

Correspondence: mvotinov@ukaachen.de and apuiu@ukaachen.de

\section{Introduction}

In everyday decision-making, we weigh the risks and rewards (i.e., probability) associated with competing outcomes in terms of relative payoffs and the potential for negative outcomes (i.e., gains vs. losses). For decades scientists have been investigating decisions under risk and ambiguity and proposed several models that quantify decision-making. Decision-making under risk typically involves a decisionmaker who knows the probability distribution of all possible outcomes associated with a decision while the outcome itself is unknown Knight (1921). Contrastingly, uncertainty reflects an unknown probability distribution and an unknown outcome. While risk and uncertainty are often treated interchangeably, discriminating between the two has been conceptually and empirically validated Ruggeri et al. (2020).

\section{Prospect Theory.}

One of the most influential frameworks for researching decision-making under risk is the prospect theory (PT, Kahneman and Tversky (1979), Tversky and Kahneman (1991). The theory posits that decisions are affected by how potential outcomes (prospects) are cognitively represented in terms of gains, losses, and their associated probabilities. As such, rational individuals aim to optimize outcomes by weighing the value, probability, and cumulative wealth. According to PT, the value $V(x, p)$ of a simple prospect that pays $x$ with probability $\mathrm{p}$ and can be represented as:

$$
V(x, p)=v(x) \omega(p)
$$

where $v(p)$ measures the subjective value of the consequence $x$, and measures the impact of probability $p$ on the attractiveness of the prospect Stanton (2017), Tversky and Kahneman (1991)

According to prospect theory, the value function $V(x, p)$ exhibits the psychophysics of diminishing sensitivity with the value function being steeper for losses (loss aversion) than for gains. In other words, a loss of $€ 50$ is felt more than a gain of $€ 50$. Loss aversion reflects a tendency to avoid losses relative to acquiring equivalent gains. The utility of monetary payoffs that depends on previous experiences is what distinguishes loss from risk aversion. Overall, prospect theory argues that decision-makers weigh gains and losses differently, weighing perceived gains more than perceived losses. This shows that humans think in terms of expected utility relative to a reference point (i.e., current financial situation/wealth) rather than in absolute outcomes.

A refinement of the original version of prospect theory, cumulative prospect theory (CPT) applies different weights to the cumulative probability distribution function instead of applying weights to the probabilities of individual outcomes Tversky and Kahneman (1992). This ensures that the value of an outcome is multiplied by a decision weight and not by an additive probability (for a model overview, please see Fox and Poldrack (2009)). A recent study across 19 countries and over four thousand participants replicated the empirical foundations of the prospect theory and showed that none of 
the prospect theory's theoretical constructs (i.e., isolation effect, framing effects, overweighting of small probabilities, etc.) from the original study were unreliable Ruggeri et al. (2020). These findings confirm that prospect theory is a robust descriptive model of decision-making under risk and uncertainty.

\section{Testosterone.}

Testosterone is one of the major steroid hormones produced in men and women (though in different quantities) that modulates brain activity by binding to intracellular androgen receptors and regulating gene expression via genomic and non-genomic effects (for a comprehensive review, see Brinkmann (2011)). It acts as neuroactive steroid by influencing ion channels or G-protein coupled receptors Höfer et al. (2013) with many of its effect being mediated by androgen receptors through aromatisation Van Honk et al. (2012). High expression of aromatase were observed in the limbic regions (with the highest activity in the amygdala, thalamus and hyppocampus) which are associated with sensory and emotion processing Biegon et al. (2010), Sasano et al. (1998). Developments in neuroeconomics and neuroendocrinology highlighted shared biological mechanisms that support decision-making involving economic risk-taking. As such, testosterone is an interesting biomarker for socioeconomic decision-making and for status seeking behaviours.

\section{Testosterone and Risk-Taking.}

Engaging in risky behaviours might be an evolutionary strategy to attain higher positions in social hierarchies where status is important. It is therefore likely that testosterone also modulates risk-taking behaviours and several studies reliably associated testosterone with social and economic decisionmaking under high risk (Apicella et al. (2014), Eisenegger et al. (2011). A recent meta-analysis showed a significant albeit small correlation between testosterone and risk-taking which was not influenced by gender nor outcome measures (Kurath and Mata (2018)). Nevertheless, there are several studies also reporting contrasting (for a review, see Mehta et al. (2015) or null results Derntl et al. (2014), Nadler et al. (2021), Stanton et al. (2021), Van der Loos et al. (2013).

Steroid hormones including testosterone preferentially modulate brain activity Dreher et al. (2007), Votinov et al. (2020) regulating the neural function of areas involved in economic decision-making and emotion regulation. Several studies measured endogenous testosterone levels and found that high levels of hormone correlate well with increased risk- taking in both men and women indicating a heightened willingness to take financial risks Chicaiza-Becerra and GarciaMolina (2017), Garbarino et al. (2011), Stanton et al. (2011a). Other studies, however, showed a positive relationship between basal endogenous testosterone levels and decreased risk-aversion thus showing that testosterone may also circumstantially promote greater risk neutrality Apicella et al. (2008), Sapienza et al. (2009), Stanton et al. (2011a). These inconsistencies are likely due to the various techniques used to determine basal testosterone concentrations. For instance, the $2 \mathrm{D}: 4 \mathrm{D}$ ratio, which has been proposed as a proxy for prenatal hormone exposure, did not correlate significantly with men's or women's risk preferences Apicella et al. (2008), Derntl et al. (2014) nor did it mediate the relationship between hair testosterone concentrations and risk-taking measured using the Balloon Analogue Risk Task (BART) Ronay et al. (2018). These results suggest potentially divergent activating and organizing effects of testosterone on brain and behavior. Other studies investigated the effects of exogenous (pharmacologically-elevated) testosterone on risky decisionmaking. In men, transdermal testosterone shifted investments towards riskier assets Cueva et al. (2015) and increased risk-taking under conditions of unknown probabilities during strategic decision-making Goudriaan et al. (2010), Wagels et al. (2017). Furthermore, exogenous testosterone increased risk-taking in the Iowa Gambling Task (IGT) in a group of women, likely by increasing reward sensitivity while decreasing punishment sensitivity. Similarly, endogenous testosterone accounted for $11 \%$ decision-making in the uncertainty phase of the IGT Singh (2021).

The literature on testosterone and risk-taking is far from conclusive. For instance, several studies Boksem et al. (2013), Zethraeus et al. (2009) found no effect of exogenous testosterone (sublingual administration) on women's risk attitudes or ambiguity tolerance. Likewise, in a double-blind withinsubjects study investigating risk-taking and loss aversion, Stanton and colleagues (2021) found no consistent relationship between pharmacologically-elevated testosterone and economic decisions Stanton et al. (2021). Instead, they argue that that findings may be explained by several situational moderators. Indeed, other studies also showed a contextdependent role of testosterone in regulating risk-taking either to promote social status Vermeer et al. (2020) or abolish loss chasing Wu et al. (2016). Moreover, data from 306,248 participants of the UK Biobank further substantiates testosterone's context-dependent effects and highlights little evidence for its causal role in socioeconomic decisionmaking or risk-taking Hughes et al. (2021). These studies show various measures directly or indirectly associated with risky decision-making that can be altered by short-term fluctuations in testosterone concentrations. Nevertheless, direct causal comparisons are limited by methodological constraints that may hamper interpretations. For instance, the doses used to pharmacologically elevate testosterone levels are heterogeneous within and between sexes (i.e., in men, exogenous applications vary from 50 to $150 \mathrm{mg}$ transdermal doses vs the standardised $0.5 \mathrm{mg}$ sublingual administration in women). Likewise, endogenous measures of testosterone levels are computed using 2D:4D ratios, saliva, blood, or hair sampling that all have inherent limitations.

\section{Testosterone and Consumerism. Endowment Effect.}

Not only is testosterone associated with risk-taking and decision-making but it also influences consumer behaviour by altering consumer-based choices and preferences. For instance, prenatal testosterone exposure correlates well with men's courtship-related gift-giving (Nepomuceno et al. 
$(2016 a, b)$ in an attempt to attain a certain status. One way to attain status is through wealth acquisition. In addition to attaining status, we also project status through the choices of what we buy and, importantly, through what we buy and display to others. We may choose goods that make us more attractive to the opposite sex or that help us outcompete or intimidate others of the same sex. For example, in men, resource accumulation may reflect status (e.g., wearable luxury goods) that can be used to enhance one's appeal to potential mates Sundie et al. (2011). Another study by Aspara and Van den Bergh (2014) examined the effects of the hormone on consumers' preferences for gendered products. They found that men with higher prenatal testosterone exposure preferred more masculine products and dressed in more masculine colors than men with lower levels of testosterone exposure Aspara and Van Den Bergh (2014). The study investigated the influence of testosterone on men's preferences for positional goods, and they found that administering testosterone increases men's preference for status brands, compared to brands of similar perceived quality but lower perceived status. Moreover, testosterone increases positive attitudes toward positional goods when they are described as status-enhancing Nave et al. (2018). In women, preferences for wearable luxury goods may be enhanced when women are threatened by other attractive women Durante et al. (2014). On the other hand, consumer behavior could influence testosterone levels as well. One study showed that participants that drove a new Porsche experienced an increase in testosterone as a result, while those who drove a twenty-year old Toyota did not (Saad and Vongas (2009)). While this interaction may indeed lead to increased testosterone levels, the effect could also be a result of noradrenergic arousal-bursts that could account for the sharp increase in testosterone level Sapolsky and Meaney (1986), Schultheiss and Stanton (2009).

Unlike risky decision-making, riskless choice relates to prospect theory through the so-called endowment effect Kahneman et al. (1991), Thaler (1980) which is the observation that individuals will preferentially sell goods at a higher price than they would purchase them for. It has been used as evidence for theories of reference-dependent preferences and loss aversion and it shows that the mini-mum amount of money people are willing to accept (WTA) when selling an item is significantly higher than the mini-mum amount they are willing to pay (WTP) for the same item if they do not already own it Ariely et al. (2005), Camerer (2011), Gächter et al. (2021), Kahneman et al. (1991), Votinov et al. (2010, 2013). Endowing a person with a good (even hypothetically), seems to establish a reference point people move away from only reluctantly, or if they are paid a large sum Camerer (2011). This observation underscores a status quo bias towards own goods. Consumer choices are thus directly linked to competition and competing interests Durante and Griskevicius (2016) which are also subject to endocrine effects Cherki et al. (2021). An extreme feeling of possession may lead to an excessive acquisition of items resulting in experiencing difficulties discarding them, which can be linked to hoarding disorder Pushkarskaya et al. (2020).
The type of goods (utilitarian vs hedonic) that consumers buy or sell heavily influence the WTA/WTP ratio. Utilitarian goods focus on function, practicality, and are generally more of what people "need" as opposed to hedonic goods, which provide more fun, are not as functional, and reflect what people "want" Hirschman and Holbrook (1982), Strahilevitz and Myers (1998). Buying and selling hedonic instead of utilitarian goods has previously been found to increase the WTA/WTP ratio Chan (2015). For example, one field survey Dhar and Wertenbroch (2000) demonstrated this by collecting data from two hundred seventeen MBA students about the features of the car they owned. Participants rated their car on a scale from one to nine for hedonic dimensions and then separately for utilitarian dimensions. They were then asked how much money they would be willing to sell their car for. As the researchers predicted, those with a more hedonic car would demand more money concerning their car's market price than those with more utilitarian cars would. Another study by Cramer and Antonides (2011) tested decision making with respect to hedonic versus utilitarian food products. They observed strong endowment effect for hedonic compared to utilitarian food products and pro-posed that status quo bias for hedonic food products may lead to relatively unhealthy food choices. A potential reason for this disparity may be because the owners of hedonic goods may develop a more significant symbolic relationship those goods than with utilitarian goods Belk (1988).

\section{Goal of the study.}

While several studies investigated how consumers experience the endowment effect and the role testosterone plays in consumerism, the interaction between consumerism and endowment effect has not yet been thoroughly explored. As such, we investigate the role of testosterone on risk-taking and loss aversion under positive and negative framing. First, we examine whether testosterone administration affects decisionmaking in a series of gambles under positive and negative framing (gain and loss) associated with various probabilities. Under testosterone administration, we expect participants to show increased risk-seeking behaviours regardless of framing type. Second, we investigate testosterone's effect on loss aversion (endowment effect) during the economic valuation of two goods categories (utilitarian vs hedonic). We hypothesise that the endowment effect (and therefore the WTA/WTP ratio) will be higher for hedonic compared to utilitarian goods. Compared to placebo, we expect testosterone administration to further increase the endowment effect (and the WTA/WTP ratio) for both types of goods. We test these hypotheses for one-tailed significance since we predicted directionality based on previous findings.

\section{Methods}

\section{Socio-demographics data.}

Forty healthy male participants with mean age $23.2 \pm 2.9$, participated in the study. These individuals concurrently took part in a larger, separate functional MRI study and agreed to 
Table 1. Game types

take part in this study additionally. Due to technical problems hormonal data was recorded for 39 participants (missing data was replaced by mean values for each group). For the Endowment task, we analysed data from 37 subjects because two participants did not complete the task while another participant's data was removed because a value of zero was indicated when selling/buying items on more than $30 \%$ of the items.

\section{Testosterone application.}

Participants performed the tasks twice separated by a washout period of a minimum of seven days. On one visit they were administered a $100 \mathrm{mg}$ colourless and odourless testosterone gel that was applied to participants suprascapular area (shoulders and lower neck) and allowed to dry for $15 \mathrm{~min}$. The testosterone gel consisted of Testotop $®$ (active testosterone ingredient), 96\% ethanol, polyacrylate and propylene glycol, disodium EDTA, trometamol, and purified water. On the second visit, they received a placebo gel containing the same ingredients as above but excluding the active testosterone ingredient (Testotop $®)$ ). Treatment order was counterbalanced and randomised and the protocol was previously validated Puiu et al. (2019). Half of the participants received the testosterone gel first and placebo second. Participants performed the task $1.5 \mathrm{~h}$ following testosterone/placebo administration so as to allow for testosterone's loading period Puiu et al. (2019). The design of the study was double-blind, meaning that neither researchers nor participants were aware what treatment was administered when. At the end of each session, we asked the participants and experimenter to state what treatment they thought was administered.

\section{Hormone Profiles.}

Testosterone and cortisol serum concentrations were analyzed by electrochemiluminescence immunoassays (ECLIA, Roche ${ }^{\circ}$ Diagnostics $\left.\mathrm{GmbH}\right)$. For testosterone, the inter-assay coefficient was 2.4 with a lower detection limit of 0.09 $\mathrm{nmol} / \mathrm{L}$. Respective inter-assay data for cortisol was 3.8 with a lower detection limit of $0.054 \mu \mathrm{g} / \mathrm{dl}$. Intra-assay coefficients for testosterone and cortisol were below 3. All analyses were conducted under strict internal and external quality control at the Clinical Chemistry, Haematology, Virology, and Microbiology Laboratory Diagnostic Centre (LDZ) of the RWTH Aachen University Hospital.

\section{Experiment design.}

The experimental setup consisted of a risk-taking and an endowment task see Fig 1

\section{Risk-taking task.}

Participants made a choice between two options where one was a sure option and another one a gamble option with different probabilities $(p)$ of winning and losing. There were five binary choices under two framings. Under positive framing, option A reflects winning a larger amount of money or getting nothing. Option $\mathrm{B}$ indexes sure winning but with a

\begin{tabular}{lccccc}
\hline & \multicolumn{2}{c}{ Positive } & \multicolumn{2}{c}{ Negative } & Probabilies \\
\hline & $\mathrm{A}($ risky) & $\mathrm{B}($ sure $)$ & $\mathrm{A}($ risky) & $\mathrm{B}($ sure $)$ & $\mathrm{P}($ risky) \\
\hline game 5 & 2000 & 1500 & 2400 & 1200 & 0.9 \\
\hline game 4 & 500 & 250 & 600 & 200 & 0.7 \\
\hline game 3 & 1000 & 500 & 1000 & 500 & 0.5 \\
\hline game 2 & 500 & 250 & 1800 & 600 & 0.3 \\
\hline game 1 & 250 & 50 & 1200 & 400 & 0.1
\end{tabular}

lesser payoff (i.e., amount of money). Under negative framing, option A reflects losing more money or not losing anything. Option B reflects a sure loss of a small amount (Figure 1, Game 1 exemplifies some questions and the options for the framing type). Each option was associated with the following probability sets: $0.1,0.3,0.5,0.7$, and 0.9 for both framings (see Table 1 for the probability distributions for all options). All games were presented in a pseudorandom order and participants played the game twice (once under placebo and once under testosterone administration).

\section{Endowment effect task.}

For this task, participants were presented with two categories of goods:

(1) hedonic goods, which can be described as items that one desires because they are attractive and bringing fun

(2) utilitarian goods, which are items that are rather practical and goal-oriented.

Photographs of all items were taken from the Amazon online shop. All items were pretested in a preliminary behavioral experiment on a separate group of participants to see if it is easy to distinguish between two categories. Each item was presented twice randomly during each session (placebo and testosterone), and participants had to estimate the items' value (in Euro). When it was indicated that they own an item, participants were asked to indicate the minimum amount of money they were willing to accept (WTA) to give it up. When the item belonged to someone else, participants were asked to indicate the maximum amount of money they were willing to pay (WTP) to acquire it.

\section{Data analysis}

We used Python (v3.8) for data preprocessing and analyses. Preprocessing and exploratory data analyses were performed with pandas (v1.2) and seaborn (v 0.11) libraries. Bayesian statistical analysis and modelling were performed with a custom code based on PyMC3 (v 3.11) (Python package for Bayesian statistical modeling and probabilistic machine learning) and openly accessible through Jupyter notebooks containing detailed research procedures, methods, and results. A detailed description of every step for each analysis with the code and data are available at GitHub and OSF. Bayesian modelling involves making choices concerning prior distributions, likelihood functions and model evaluation, which includes posterior checks and quality assessments Aczel et al. (2020). Here we provided and briefly described the models we used for data analysis. Full model 


\section{GAME 1 (Risk-Taking)}

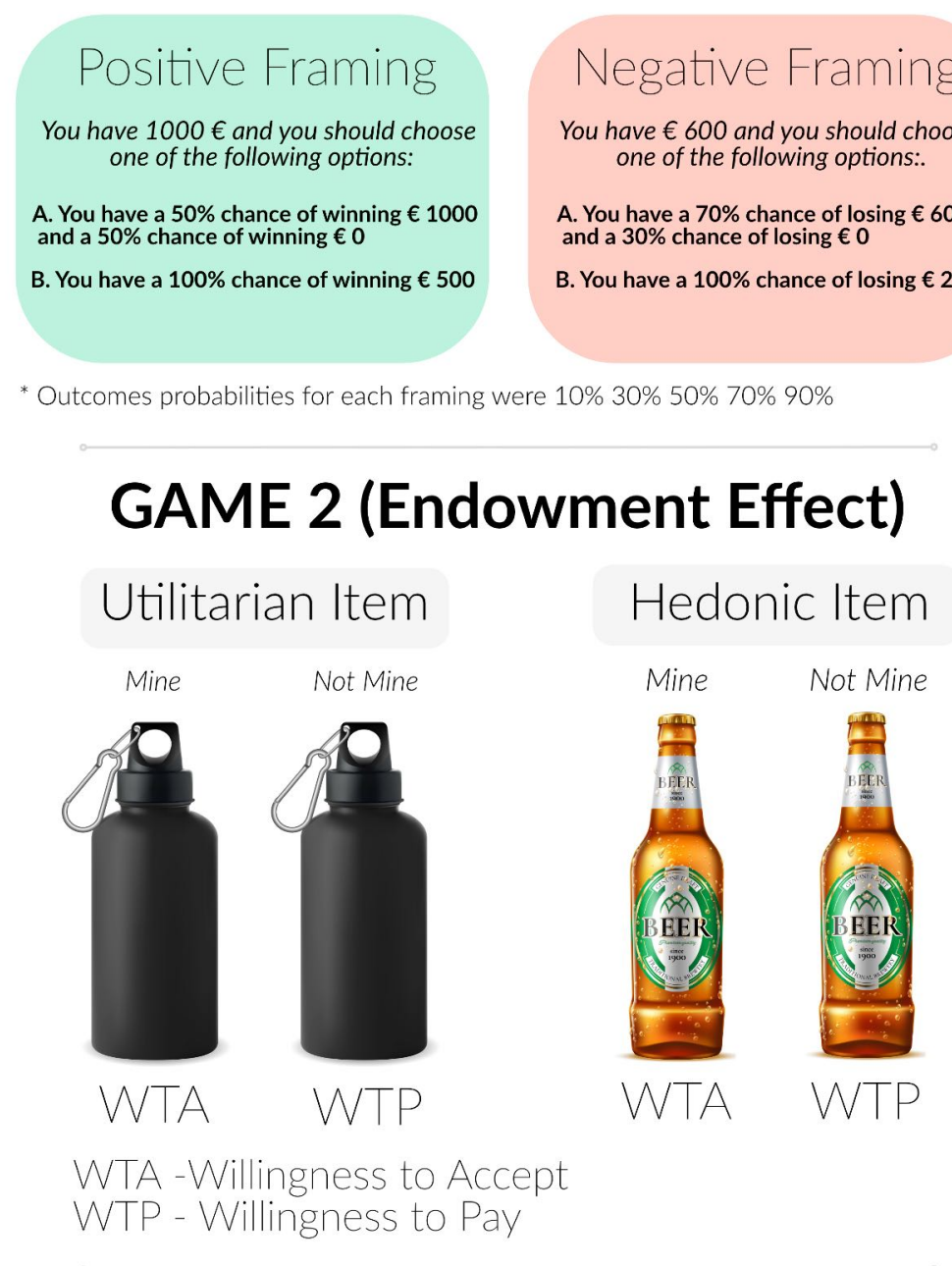

Fig. 1. Experimental design of 2 games.

specification and the description of estimation procedures are available in the supplementary file.

\section{Endowment effect exploration.}

We used standard hypothesis testing and Bayesian modelling to investigate the endowment effect. The following hypotheses were tested:

\section{Endowment effect regardless of the type of goods}

Formally:

- H0: $W T A / W T P=1$ against

- $\mathrm{H} 1: W T A / W T P>1$

A one-tailed t-test was performed to check this hypothesis. In addition, for each item we modelled a $W T A / W T P$ ratio using multilevel Bayesian regression (McElreath (2018), Nilsson et al. (2011)). There are two cluster variables in this experiment: item and participant. In other words, each observation has an item and a participant indicator. To model this, we constructed a group-level hyperparameter describing the overall average WTA/WTP ratio $\left(\mu_{0}\right)$ with two varying intercept parameters responsible for variations through goods $g_{\text {item }}$ and subjects $\mu_{\text {ind }}$. Since the WTA/WTP value is strongly positive, we used a logarithmic link function, so that the parameters $\mu_{0}, \mu_{\text {ind }}, g_{\text {item }}$ covered the entire real line:

$$
\log \left(\mathrm{WTA} / \mathrm{WTP}_{[\text {ind,item }]}\right)=\mu_{0}+\mu_{\text {ind }}+g_{\text {item }}
$$

As a result, we were able to estimate the endowment ratio for each item as a posterior distribution for $\exp \left(\mu_{0}+g_{\mathrm{item}}\right)$. Full model specification with the quality assessment is provided in supplementary material and in source code.

2. Differences between hedonic and utilitarian items. Afterwards, we tested the differences between hedonic and utilitarian goods to examine whether hedonic items had significantly higher WTA/WTP ratio than utilitarian items. 
- H0 : $\mathrm{WTA} \mathrm{WTP}_{h}=\mathrm{WTA}_{\mathrm{WTP}}$

- H1: $\mathrm{WTA} \mathrm{WTP}_{h}>\mathrm{WTA} \mathrm{WTP}_{u}$

This hypothesis was tested with a one-sided t-test. We also estimated the posterior distribution for the hedonic and utilitarian WTA/WTP ratio using type of goods as a cluster variable, accounted for by the following model:

$$
\log \left(\mathrm{WTA} / \mathrm{WTP}_{\text {type }}\right)=\mu_{\text {type }}
$$

3. Differences between testosterone and placebo We used repeated-measures analyses of variance (ANOVA) to examine the differences between testosterone and placebo groups and modelled individual item effects using a Bayesian model. We used the same model as the one described above and introduced a testosterone-related variable. As a result, the logarithm of WTA/WTP ratio was modelled as the sum of the following parameters: group-level ratio $\mu$, testosteronerelated coefficient $b$ proportional to the level of hormone changes $T_{\text {change }}$, individual variation for each participant $\mu_{\text {Ind }}$ and item variation $g_{\text {goods }}$

$$
\log (\mathrm{WTA} / \mathrm{WTP})=\mu_{0}+b * T_{\text {change }}+\mu_{\text {ind }}+g_{\text {item }}
$$

\section{Risk-taking under framing.}

The generally accepted model of people making decisions under risk is based on the cumulative prospect theory Tversky and Kahneman (1992). According to prospect theory Fox and Poldrack (2009), making a choice between two gambling options is based on a subjective value that represents its desirability to the decision maker. The subjective value of money for option $\mathrm{O} V(O)$, where option $\mathrm{O}$ is payoff $x$ with probability $p$ is given by (1). The subjective value of payoff is defined as a power function from payoff:

$$
v\left(x_{i}\right)= \begin{cases}x^{\alpha}, & \text { if }(x)>0 \\ -\lambda(-x)^{\alpha}, & \text { else }\end{cases}
$$

This form of value function exhibits the psychophysics of diminishing sensitivity to the value of payoff. The weighting function captures the diminishing sensitivity to changes in probability. Diminishing sensitivity implies an inverse sshape weighting function that is concave near zero and convex near one. This shape reflects the observed risk attitudes with underweighted high probabilities and overweighted low probabilities. Here we used a single parameter function as suggested in Tversky and Kahneman (1992).

$$
\omega(p)=\frac{p^{c}}{\left[p^{c}+(1-p)^{c}\right]^{1 / c}}
$$

According to the prospect theory, a rational decision maker should always choose an option with a larger subjective value. To include possible errors caused by noise, cognitive states or other factors, an additional sensitivity parameter $\phi$ is introduced to the choice model. A 0 to 1 choice probability is usually described by a sigmoid transformation. Finally, the choice probability transforms in the following way:

$$
p(A, B)=\frac{\mathrm{e}^{\phi V(A)}}{\mathrm{e}^{\phi V(A)}+\mathrm{e}^{\phi V(B)}}=\frac{1}{1+\mathrm{e}^{-\phi(V(A)-V(B))}}
$$

In case of equal options, we have a random choice with a 0.5 probability. When option A has a bigger subjective value, the expression $\mathrm{e}^{-\phi(V(A)-V(B))}$ tends to zero and the probability to choose option A tends to 1 . According to the previous studies, the typical value for $\alpha$ lies in ranges [0.75, 0.95] and for $c-[0.6,0.96]$ Fox and Poldrack (2009).

\section{Modification of CPT model to hormone dependent risk- taking framing paradigm.}

To model risk-taking and choice behaviour in our framing paradigm, we used a Bayesian approach for several reasons. First, Bayesian modelling allows us to estimate prior knowledge about decision-making processes. Second, the output of a Bayesian model is an interpretable parameter with clear posterior distributions that may be used as an alternative to $\mathrm{p}$ values. Last, there are several studies where hierarchical Bayesian approach for CPT was successfully applied Nilsson et al. (2011, 2020), Shiffrin et al. (2008).

The choice rule (see eq. 4) may be rewritten as a general linear model with the logit link function (inverse to logistic function) McElreath (2018):

$$
\operatorname{logit}(p)=\phi * \operatorname{gap}(A, B, \alpha, c),
$$

where $\alpha, c$ - value function and choice probability correction from CPT theory parameters and $\operatorname{gap}(A, B, \alpha, c)=$ $V(A, \alpha, c)-V(B, \alpha, c)$

We introduced an intercept to the choice rule because, in practice, considering equal subjective value, a sure option is more often preferred over a risky one. The modification with the intercept can be mathematically expressed as follows:

$$
\operatorname{logit}(p)=\operatorname{Intercept}+\operatorname{Beta} * \operatorname{gap}(A, B, \alpha, c)
$$

In previous studies Fox and Poldrack (2009) the differences between the options (gap) ranged very little (up to $10 \$$ ). Our experimental setup entailed range changes between 250 and 1200 Euro. To reduce scale sensitivity, we used $\log 1 p$ transformation for the gap:

$$
\log \mathrm{Gap}=\operatorname{sign}(\text { gap }) \log (1+\operatorname{abs}(\text { gap })))
$$

With this transformation, small gap values remain approximately the same because $\log (1+x) \sim x$ and big values are diminished. We suggest that both framing options $(F)$ have their own Intercept and Beta parameters. The final model for choice rule was estimated using

$$
\operatorname{logit}(p)=\operatorname{Intercept}_{F}+\operatorname{Beta}_{F} * \log \operatorname{Gap}\left(A_{F}, B_{F}, \alpha, c\right),
$$

where Intercept and Beta depend on the serum testosterone level in the following way:

$$
\left\{\begin{array}{l}
\text { Intercept }=a_{F}+\delta \_a_{F} * \text { Tchange, } \\
\text { Beta }=b_{F}+\delta \_b_{F}
\end{array}\right.
$$


where Tchange indicates the change in testosterone level. Participants took part in both framings and each hormone condition, so Intercept (shift to sure option) may correlate with the slope Beta (sensitivity to the gap). Moreover, since both sets of the parameters are connected to risk attitudes, we estimated a hierarchical model with covariance between all parameters. More information is provided in supplementary.

\section{Prospect theory parameters re-estimation.}

In the current experiment, we had five measurements for each individual in each framing. The primary aim of this study is not to explore prospect theory parameters but to examine the testosterone application effect on risk-taking in different framing. To simplify, we assumed that CPT parameters have no dependence on hormonal application. Thus, we estimated CPT parameters $\alpha, c$, based on data from previous decisionmaking studies. The closest experimental setup to ours that had an open dataset available was described and analysed in Nilsson et al. (2011). We replicated the hierarchical model suggested in the original paper but only for pure gain and pure loss (positive and negative framing) games, as we did not have mixed games in our study. After that we estimated the model with log transform modification and used the estimated parameters $\alpha$ and $c$ in our choice model. The results of the replication study with and without log transform modification are available in the supplementary materials and accompanying code.

\section{Results}

\section{Testosterone concentrations.}

The repeated-measures ANOVA revealed significant main effects of treatment $\left(\mathrm{F} 1,38=64.99, \mathrm{p}<.001, \eta^{2}=.63\right)$ and time $\left(\mathrm{F} 1,38=84.21, \mathrm{p}<.001, \eta^{2}=.69\right)$, as well as a significant treatment-by-time interaction effect $(\mathrm{F} 1,38=106.22$, $\mathrm{p}$ $\left.<.001, \eta^{2}=.74\right)$, indicating that testosterone levels change differently across time with transdermal testosterone relative to placebo treatment. A post-hoc analysis showed that serum testosterone concentration did not differ significantly between transdermal testosterone and placebo at baseline ( $\mathrm{p}$ $=.9$ ). However, serum testosterone levels significantly increased $(\mathrm{p}<0.001)$ in following testosterone administration at time point 1 (cca $90 \mathrm{~min} ; T 1=33.76 \pm 11.9)$ compared to baseline $(T 0=18.53 \pm 6.04)$. There was no difference $(\mathrm{p}=.58)$ in the placebo condition between baseline $(T 0=18.69 \pm 5.44)$ and $\mathrm{T} 1(T 1=19.0 \pm 6.38)$, see Fig 3. To demonstrate individual changes of testosterone level we plotted the Fig. 2.

\section{Cortisol concentrations.}

The repeated-measures ANOVA revealed significant main effects of time $\left(\mathrm{F} 1,38=166.66, \mathrm{p}<.001, \eta^{2}=.81\right)$, indicating the known circadian fluctuation in cortisol secretion. There was no effect for treatment $(\mathrm{F} 1,38=1, \mathrm{p}=$ $\left..32, \eta^{2}=.03\right)$ nor a treatment-by-time interaction $(\mathrm{F} 1,38$ $\left.=.16, \mathrm{p}=.69, \eta^{2}=.001\right)$. Post-hoc analyses showed that serum cortisol concentration did not differ significantly between testosterone $(T 0=427.30 \pm 97.87)$ and placebo treat-

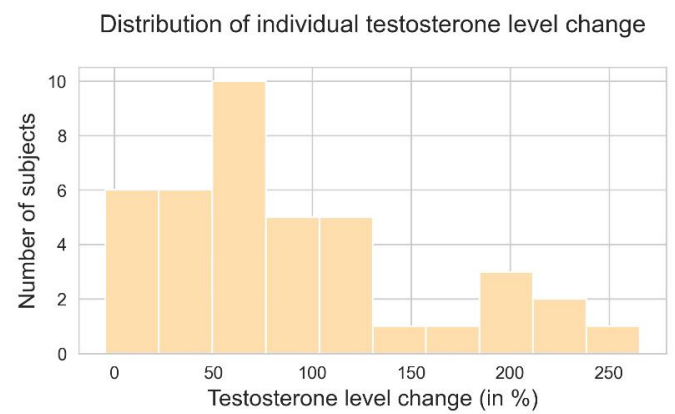

Fig. 2. Distribution of individual testosterone level change across whole sample.

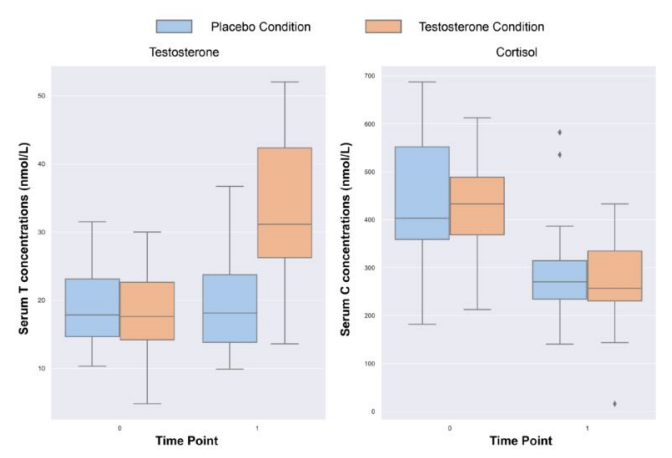

Fig. 3. Serum level for Testosterone and Cortisol for 2 time points.

ments $(T 0=441.68 \pm 120.25)$ at baseline (T0) and as well as at $\mathrm{T} 1$, where cortisol level in the testosterone condition was $T 1=273.74 \pm 80.67$ and in the placebo condition was $T 1=282.65 \pm 88.12$. Cortisol levels between baseline and T1 were significant for both treatments ( $p<.001$, see Fig. 3 ).

\section{Endowment effect.}

First, we examine the endowment effect regardless of the type of goods. Participants placed the WTA price higher than the WTP price for the same items even though they were aware the endowment of the product was not real. The results of one-sample $t$ test confirmed that the WTA/WTP ratio for all items was significantly higher $(p<0.001$, Bayes Factor $1.298 \mathrm{e}+23$ ) than for the condition without effect (WTA/WTP =1). The WTA/WTP ratio for each item in the hedonic and utilitarian category in the placebo condition is plotted in Fig.4. These values were computed through Bayesian general linear model described in methods as WTA/WTP $[i]=$ $\exp \left(\mu_{0}+g_{\text {goods_[i }}\right)$.

\section{Differences between hedonic and utilitarian items.}

We examined the differences between the hedonic and utilitarian categories and plotted the posterior distribution of WTA/WTP for both categories in Fig.5. The differences between means posterior distributions indicate that we have substantial evidence that the WTA/WTP ratio is higher for hedonic compared to utilitarian goods.

\section{Differences between testosterone and placebo.}

Here we examined if testosterone administration altered the endowment effect and if it affected both goods categories. After running a Markov Chain Monte Carlo sampler for the 


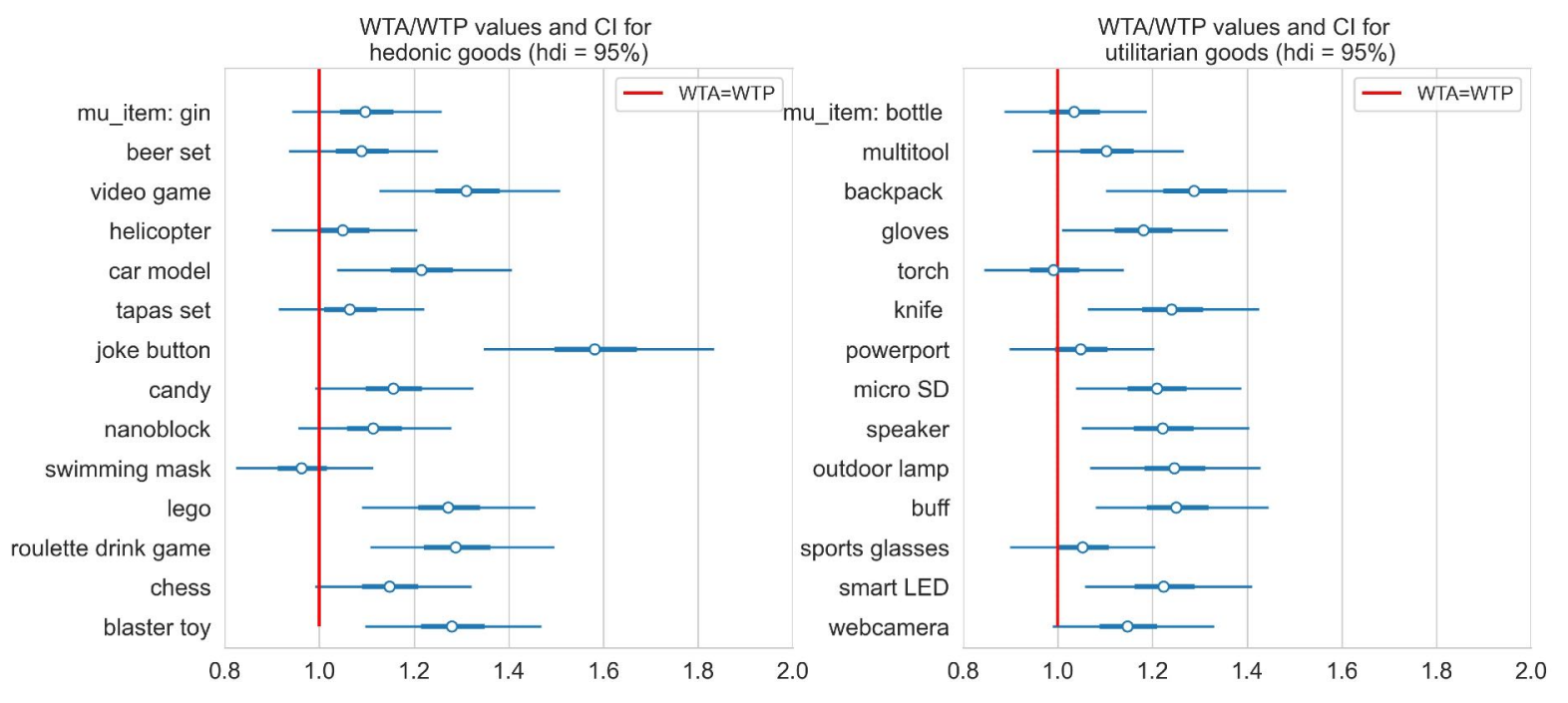

Fig. 4. The WTA/WTP ratio for each item in the hedonic and utilitarian category in the placebo condition.

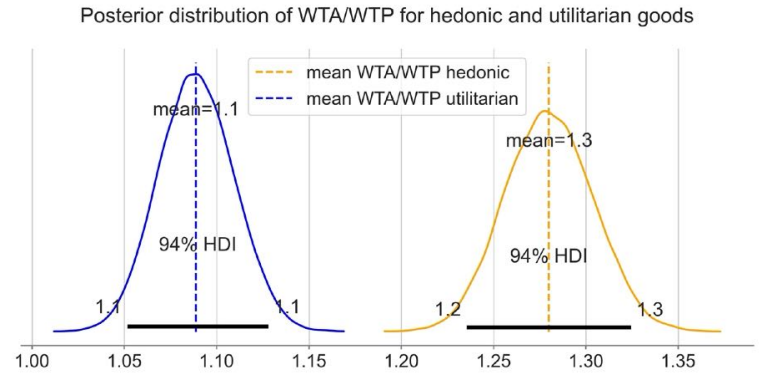

Fig. 5. The differences between means posterior distributions for hedonic and utilitarian items

model described in the methods, the posterior distribution for each item testosterone-related shifts were approximated and high density intervals containing $95 \%$ of probability were plotted in Figure 6. While we observed slight testosteronerelated shifts for some items in both categories, we did not find significant differences between the two treatment groups. Overall, we observed an endowment effect for all items regardless of their category. The endowment effect, however, was stronger for hedonic compared to utilitarian items.

\section{Risk-taking under framing.}

Participants chose between a risky and a sure (risk-free) option (see Table 1). Figure 7 shows the subjective difference between risky and sure options for each game calculated as the difference between the value function for each game as defined by the CPT equation. For computation, we used one parameter $\alpha=0.75$ and set $c=[0.7,0.8,0.9]$ for close to what has been reported in several studies (for review of these studies see Fox and Poldrack (2009). Average of all choices made by participants, grouped by games, is plotted in blue for the placebo and in orange for testosterone. The CPT parameters $c_{F}$ and $\alpha_{F}$ we re-estimated from open external dataset (Nilsson et al. (2011)), with the log transform modification of game differences. The fitted replication model is available at GitHub and OSF. As a result, we used $\alpha_{\text {Positive }}, \alpha_{\text {Negative }}=$ $0.67,0.96$ and $c_{\text {Positive }}, c_{\text {Negative }}=[0.82,0.87]$ as input param- eters for the value function calculation.

\section{Hierarchical model results.}

The hierarchical model with covariates described in the methods was estimated for recovering testosterone application effect. To verify, we estimated several additional models: pooled model (no individual differences) without testosterone parameters, pooled model with testosterone, hierarchical models with and without covariates (see in accompanying code). Adding testosterone-related parameters improved the model quality and the winning model in terms of information criterion was the hierarchical model with covariates. ROC-AUC scores scoring 1 highlight a perfect model, while random guesses yield an ROC-AUC score of 0.5. Four our baseline pooled model without testosterone-related parameters we obtained a score of 0.76 which improved when using a hierarchical model (0.94). The posterior means and standard deviations for this model parameters along with credible intervals represented in Table 2. Additionally, we provided "odds" ratio or probability of event divided by probability of no event for each parameter. It shows how the outcome changes when one of the dependent variable is changed by 1 unit. Odds equal to 1 means equal chance for both outcome. The shift parameters responsible for preference for a risk-free option are strongly negative in both framings ( $94 \%$ credible interval doesn't contain zero value). Odds for both parameters far less than 1, what means that at in case of equal options (gap $=0)$ and baseline testosterone level risk-free option is strongly preferable. Second, the beta parameter or sensitivity to gain, was positive in each framing. Third, under positive framing, testosterone-related change in sensitivity was also positive (94\% posterior credible interval doesn't contain zero; this is a reasonable amount of evidence that there is testosterone-related effects in the decisionmaking process). Forth, under negative framing, there was a testosterone-related shift to the risk-free (sure) option. Although the effect is small and credible interval contains zero, most of the probability mass is concentrated in the negative 

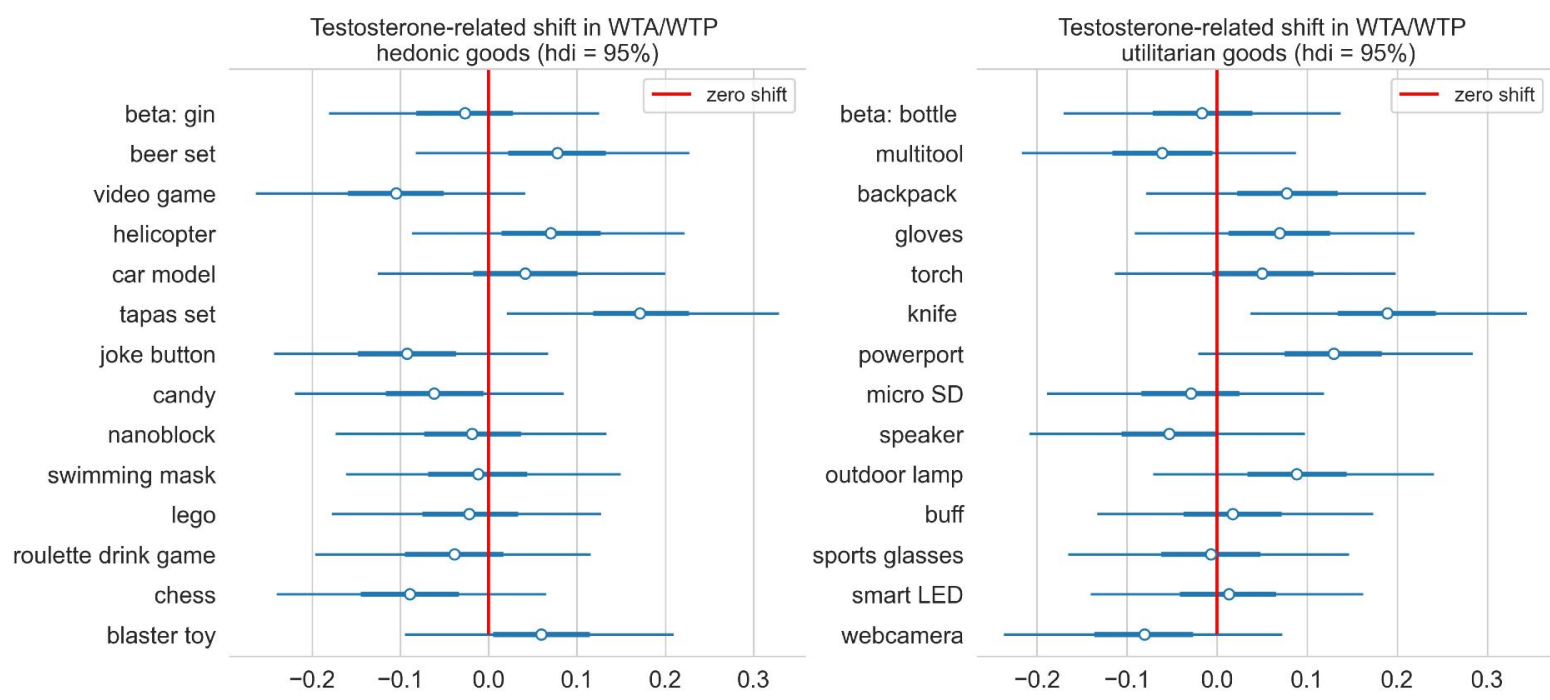

Fig. 6. Testosterone-related single item shift int WTA/WTP, grouped by type of goods. 95\% credible interval for shift for every single item contains zero (marked with red line) Table 2. Summary of the main results of multi-level Bayesian model.

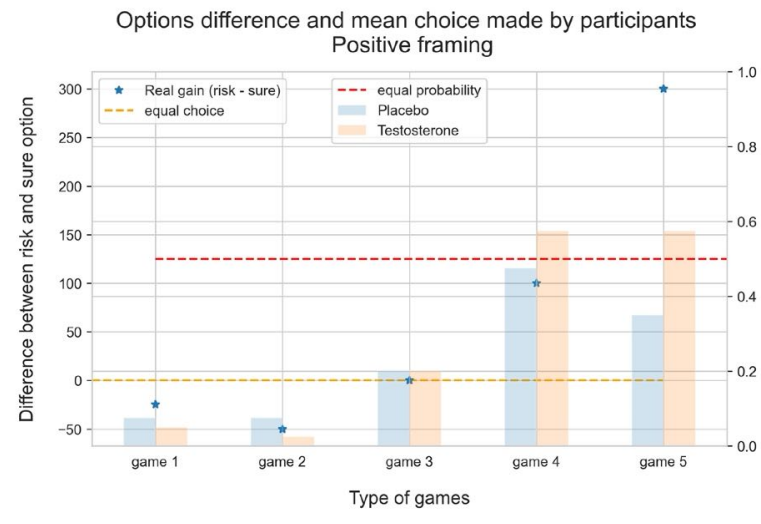

Options difference and mean choice made by participants Negative framing

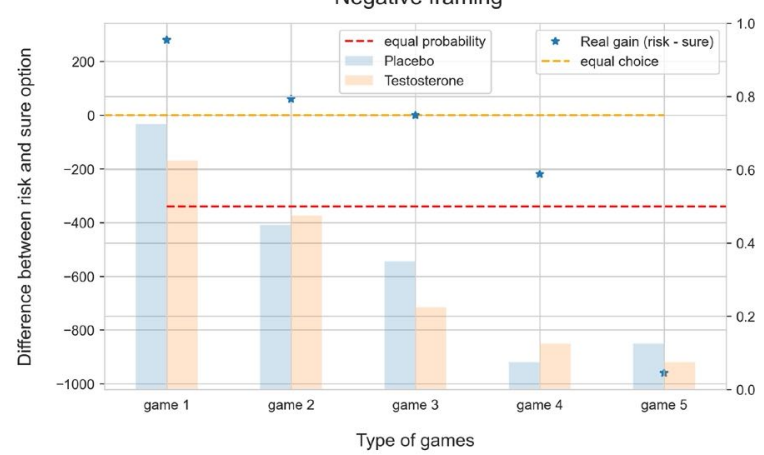

Fig. 7. Subjective difference between risky and sure options for each game calculated as the difference between the value function for each game as defined by the CPT equation. The asterisks with different colours indicate subjective differences computed for parameters $\alpha=0.75$ and set $c=[0.7 ; 0.8,0.9]$. Share of risky choices made by participants plotted with the bars. From this figure we notice several properties: (1) The difference between choices is sensitive to parameter $c$ (used in weighing probability function), specifically for several game options in positive framing. It even led to reversal choices in several cases; (2) Even when the risky option is much more favorable, willingness to risk stays at a level much lower than theoretically predicted; (3) In this set of games, the value range for subjective option differences lies in interval $[-60,60]$, which corresponds to $\left[10^{-27}, 10^{27}\right]$ in exponential scale. This leads to very low sensitivity coefficient and computational difficulty during estimation.

\begin{tabular}{cccccc} 
& mean & sd & odds & hdi 3\% & hdi 97\% \\
\hline $\begin{array}{c}\text { shift [0] (intercept) } \\
\text { Negative framing }\end{array}$ & -2.10 & 0.49 & 0.12 & -3.02 & -1.20 \\
\hline $\begin{array}{c}\text { shift [1] (intercept) } \\
\text { Positive framing }\end{array}$ & -0.98 & 0.31 & 0.38 & -1.59 & -0.41 \\
\hline $\begin{array}{c}\text { beta [0] } \\
\text { Negative framing }\end{array}$ & 0.63 & 0.14 & 1.87 & 0.37 & 0.89 \\
\hline $\begin{array}{c}\text { beta [1] } \\
\text { Positive framing }\end{array}$ & 0.63 & 0.12 & 1.87 & 0.44 & 0.86 \\
\hline $\begin{array}{c}\text { delta shift [0] } \\
\text { Negative framing }\end{array}$ & -0.44 & 0.32 & 0.64 & -1.02 & 0.18 \\
\hline $\begin{array}{c}\text { delta shift [1] } \\
\text { Positive framing }\end{array}$ & 0.24 & 0.23 & 1.27 & -0.22 & 0.66 \\
\hline $\begin{array}{c}\text { delta beta [0] } \\
\text { Negative framing }\end{array}$ & -0.02 & 0.09 & 0.98 & -0.19 & 0.14 \\
\hline $\begin{array}{c}\text { delta beta [1] } \\
\text { Positive framing }\end{array}$ & 0.27 & 0.13 & 1.30 & 0.01 & 0.52
\end{tabular}

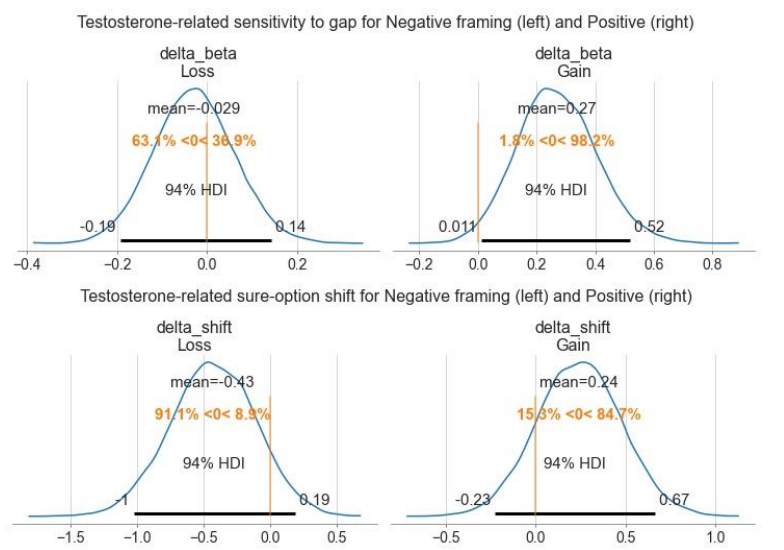

Fig. 8. The posterior distribution for testosterone-related parameters with the zero effect indicator and 94\% highest density interval (HDI)

part. The posterior distribution for testosterone-related parameters with the zero effect indicator and 94\% highest density interval is plotted in Fig 8. 


\section{Exploratory analysis and modeling of a casual relationship between changes in serum testosterone level and risky be- haviour.}

We plotted several counterfactual plots based on the estimated posterior distribution of parameters to explore a potential casual relationship between the change in testosterone level and risky behaviour. Figure9 highlights how the probability of choosing risk depends on fluctuations in testosterone levels. We fixed the difference in value function between risk and sure option at three levels: small (1 euro), medium (15 euro), and high (150 euro), and plotted dependencies for each level. The model shows that changes in testosterone levels shift the decision to be more risky under positive framing, particularly in small and medium levels. Under negative framing, increasing levels of testosterone have the opposite effect and decrease the probability to chose a risky option, especially for medium and high levels.

In the second counterfactual simulation, we modeled the effect of three fixed testosterone levels (0, 60, and 200\%) and explored risk-sensitivity to different gains. The model showed a steep shift toward riskier options under positive framing when the testosterone level increased. This further depended on the gain at stake. Under negative framing, the model also predicted an increase in risky choices when the potential loss' value increases (Fig.10). However, an increase in serum testosterone concentration diminished risk seeking.

\section{Discussion}

Using frequentist and Bayesian modelling approaches, this study investigated the effects of testosterone administration on risk-taking decision-making under positive and negative framing and the role of testosterone in the endowment effect. Treatment manipulation was successful and we observed a significant increase in serum testosterone concentrations $1.5 \mathrm{~h}$ after transdermal application. Compared to placebo, testosterone administration increased risk-taking under the positive framing and decreased under the negative framing. Our model showed that decision-making is jointly influenced by testosterone and the trade-off between gains and losses. Moreover, we observed the expected endowment effect which was more pronounced for hedonic than for utilitarian goods. Unlike our predictions, however, testosterone did not alter the endowment effect.

\section{Endowment effect.}

In the endowment task participants state their monetary preference for buying and selling various items falling into hedonic or utilitarian categories. Results showed that the WTA/WTP ratio was significantly higher and thus we observed the predicted endowment effect. The finding aligns with previous literature Carmon and Ariely (2000), Gächter et al. (2021), Kahneman et al. (1991), Knetsch (1989), Thaler (1980), Van Dijk and Van Knippenberg (1998), Votinov et al. (2013) that demonstrated an endowment effect across various types of goods (real owned and imaginary) as well for different types of transactions (goods-to-money, goods-to-goods).
Moreover, as predicted, we found a larger endowment effect for hedonic compared to utilitarian items. Previous findings argued that owning hedonic items might facilitate the development of a symbolic relationship to the items compared to items serving a clear purpose (i.e., utilitarian; Belk (1988)). This emotional attachment towards an item can be associated with more positive emotions and increased aesthetic value which might resemble the feelings hedonic items yield (Ariely et al. (2005)).

Testosterone administration did not alter the endowment effect in general nor did alter the preference for goods. Investigating items individually, we found an increase in WTA/WTP ratio only for a few of the items in both groups. We believe this effect could be item specific and bound to the subjective value individuals ascribed to them or, alternatively, could be random noise. Previous studies found that the effect of testosterone on consumer behaviour was associated with status or owning luxury goods that served to attract mates, intimidate potential rivals (Nepomuceno et al. (2016a,b)) or change product preferences Aspara and Van Den Bergh (2014). However, Wu and colleagues (2017) tested the association between competition, testosterone fluctuation, and conspicuous consumption and found no evidence for a hormonal regulation of these effects $\mathrm{Wu}$ et al. (2017). Nevertheless, winners in the competition showed increased selfreported WTP and an implicit bias towards high-status products. These studies, however, measure testosterone levels predominantly using prenatal markers (i.e., 2D:4D ratio) that have limited ecological validity Berenbaum et al. (2009), Nadler et al. (2021). Thus one cannot rule out the situational effects of decision-making that may be additive to increased endogenous testosterone concentrations. Future research should address the association between the type of consumer goods and test real items that participants own while controlling for their emotional attachment to a given item (e.g., childhood toy or present from a beloved one).

\section{Risk-taking.}

There is ongoing debate as to whether testosterone increases risky behaviour and choice. Our aim was to examine whether testosterone administration affects risky behaviour in a series of games under positive and negative framing (gain and loss) that were associated with different probabilities. We expected participants to show increased risk-seeking behaviours regardless of framing type but we only observed this pattern in the positive framing condition.

Several studies showed a causal or correlational effect of endogenous and exogenous testosterone on increased risktaking behaviours. For instance, high endogenous testosterone levels correlated with increased risk-taking in both genders and with the willingness to take higher financial risks Chicaiza-Becerra and Garcia-Molina (2017), Garbarino et al. (2011). Similarly, baseline endogenous testosterone levels were associated with increased risk-taking Stanton et al. (2011a) showing that both women and men with higher testosterone levels acted riskier than the groups having lower testosterone levels. Similar results were observed in stud- 

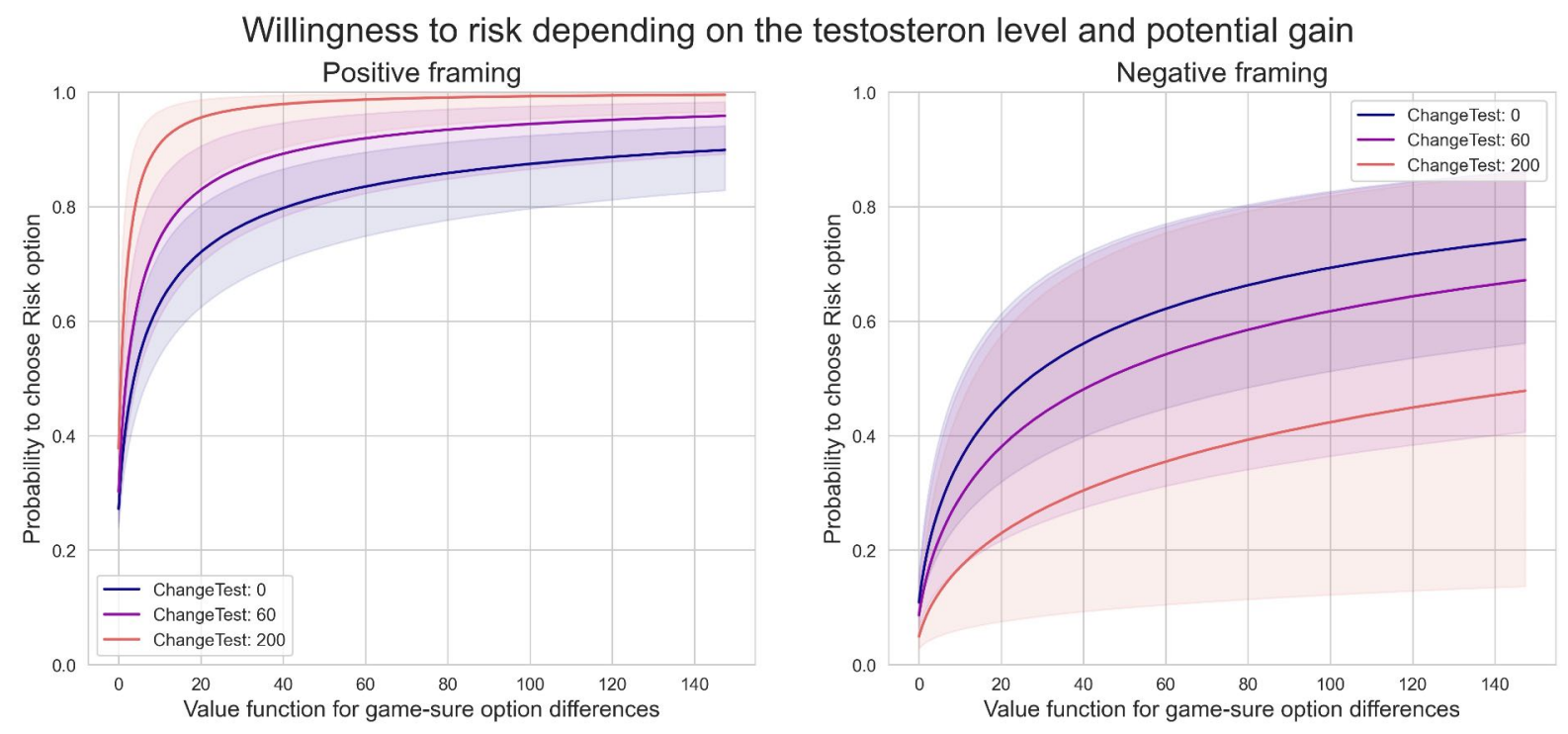

Fig. 9. Probability of choosing risk depends on potential gain

Willingness to risk depending on the testosteron level and potenial gain
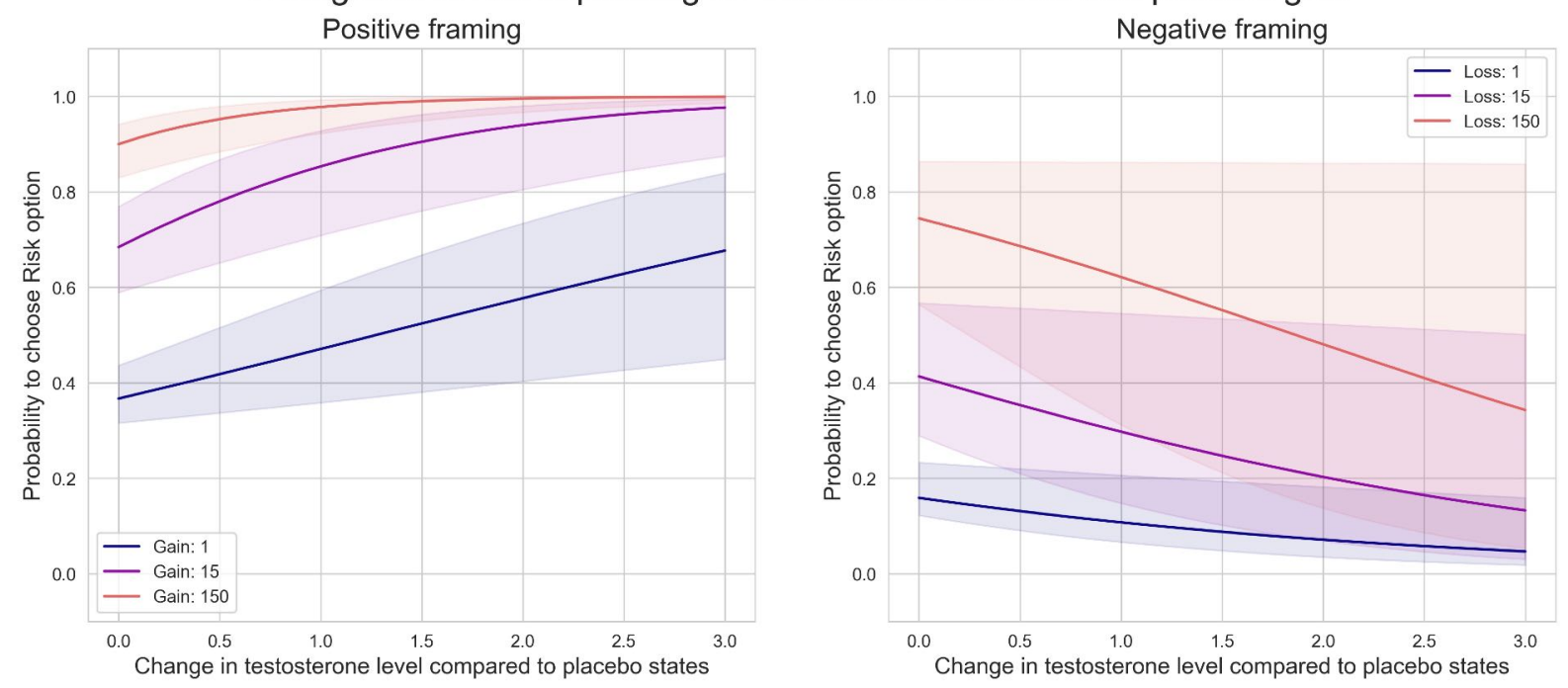

Fig. 10. Probability of choosing risk depends on testosterone level

ies that altered testosterone levels pharmacologically. For instance, exogenous testosterone increased risk-taking in the Iowa Gambling Task van Honk et al. (2004) by preferentially modulating the punishment and reward sensitivities. Another study using the Iowa Gambling Task showed a joint role of cortisol and testosterone in decision-making in the Singh (2021). Specifically, cortisol impaired decisionmaking in trials with high uncertainty, while testosterone improved decision-making in high risk trials. Several other studies examined the effect of testosterone using the Balloon Analog Risk Task (BART). Goudriaan et al. (2010) examined the effects of testosterone after treating participants with an aromatase inhibitor (letrozole $2.5 \mathrm{mg}$ ) for a week and found increased risk-taking in the high testosterone group in the BART but not in the Iowa Gambling Task Goudriaan et al. (2010). Moreover, Wagels and colleagues (2017) found an interaction between MAOA polymorphism and testosterone and showed that MAOA-S carriers played riskier than the
MAOA-L carriers Wagels et al. (2017).

Only a handful of studies used a similar approach to ours in testing risk-taking behaviours using binary decision games. Stanton et al. (2011b), for instance, measured testosterone levels in participants who performed a risk preference, loss aversion, and ambiguity task and found a U-shaped effect for risk and ambiguity. In other words, participants with low and high testosterone levels were risk- and ambiguityneutral, whereas individuals with intermediate testosterone levels were risk- and ambiguity-averse. These findings contradict our results where we observed increased risk-taking behaviour under positive framing. Our model showed that the shift to risky behaviour depends on the individual level of testosterone fluctuation, the gain at stake, and the probability of obtaining this gain. We observed a reverse behavioural pattern under negative framing. Contrary to our predictions, testosterone administration tuned participants' behaviour to be more risk-averse. Regardless of treatment (placebo or 
testosterone), however, participants were more risk-taking when the prospected financial gains were higher. As such, we suggest that participants follow an individual outcome optimisation strategy and reduce potential loss when inevitable while increasing potential gains when there is a chance for it. This optimisation strategy, however, is less susceptible to hormonal influence and more in line with contextual factors. Nadler et al. 2021 (preprint) presented participants with two gambles under positive framing. Participants could select either a sure option to get $\$ 1$ or gamble with obtaining $\$ 3$ with a varying probability between 0.1 and 1 or getting nothing Nadler et al. (2021). They excluded from analysis participants who showed intransitive preferences and classified the remaining participants into three risk-taking levels: risk-averse, slightly risk-averse, and risk-loving. Their analyses revealed similar risk-taking profiles and no treatment effects. Another recent study by Stanton et al. (2021) similarly found no consistent relationship between testosterone and economic decision-making. Since testosterone does not work in isolation, it is likely that potential contextual moderators may alter the circumstances under which testosterone affects economic decision-making.

\section{Limitation}

Our results should be interpreted in the light of several limitations. Only young, healthy men took part in this study. Therefore interpretations should be made with caution. Moreover, due to inherently different hormonal profiles, the results cannot be generalized to women. Nevertheless, prior animal model and human research suggests that acute testosterone effects are expected to manifest predominantly in men due to the distinct hormonal profiles. Last, we did not have an endogenous measure of testosterone concentration prior to the testing sessions (2D:4D measurement) to correlate with baseline intake testosterone levels. There is evidence that prenatal testosterone plays a strong role in the activational effects of testosterone for higher-order social cognition Terburg et al. (2016). Nevertheless, 2D:4D ratio results regarding testosterone effects are rather heterogenous and susceptible to measurement errors/bias.

\section{Conclusions}

This study showed the effect of testosterone administration on risk-taking behaviour in young healthy men. Testosterone increased risk-taking behaviours under positive framing and risk aversion under negative framing. However, the sensitivity to gain remained positive in each framing. We found evidence for the endowment effect which was increased for hedonic compared to utilitarian items. This effect was independent of testosterone. Our results underline the modulatory role of testosterone on risk-taking and the models we tested in the current study can be used a predictive models. For instance, if further evidence finds a robust effect of testosterone administration on economic decision-making, these findings may be of use to men presently self-medicating with androgen supplements. Given that risk tolerance is likely altered by testosterone (endogenous or exogenously manipulated), managerial implications are paramount.

\section{ACKNOWLEDGEMENTS}

Acknowledgments:

Andrei A. Puiu was supported by a scholarship from the International Research Training Group-The Neuroscience of Modulating Aggression and Impulsivity in Psychopathology (IRTG-2150) of the German Research Foundation (DFG-Projektnummer 269953372/GRK2150).

Conflict of interests:

The authors declare no conflicts of interest.

Contributions:

M.V. and A.A.P. designed the experiment., A.A.P performed the experiment, I.K, and M.V. analyzed the data and M.V.,I.K. and A.A.P. wrote the manuscript. M.V.,I.K., U.H., K.K., and A.A.P revised the manuscript and provided critical feedbacks.

\section{Bibliography}

B. Aczel, B. Szaszi, A. Sarafoglou, Z. Kekecs, Š. Kucharskỳ, D. Benjamin, C. D. Chambers, A. Fisher, A. Gelman, M. A. Gernsbacher, et al. A consensus-based transparency checklist. Nature human behaviour, 4(1):4-6, 2020.

C. L. Apicella, A. Dreber, B. Campbell, P. B. Gray, M. Hoffman, and A. C. Little. Testosterone and financial risk preferences. Evolution and human behavior, 29(6):384-390, 2008.

C. L. Apicella, A. Dreber, and J. Mollerstrom. Salivary testosterone change following monetary wins and losses predicts future financial risk-taking. Psychoneuroendocrinology, 39:58-64, 2014.

D. Ariely, J. Huber, and K. Wertenbroch. When do losses loom larger than gains? Journal of Marketing Research, 42(2):134-138, 2005.

J. Aspara and B. Van Den Bergh. Naturally designed for masculinity vs. femininity? prenatal testosterone predicts male consumers' choices of gender-imaged products. International Journal of Research in Marketing, 31(1):117-121, 2014.

R. W. Belk. Possessions and the extended self. Journal of consumer research, 15(2):139-168, 1988.

S. A. Berenbaum, K. K. Bryk, N. Nowak, C. A. Quigley, and S. Moffat. Fingers as a marker of prenatal androgen exposure. Endocrinology, 150(11):5119-5124, 2009.

A. Biegon, S. W. Kim, D. L. Alexoff, M. Jayne, P. Carter, B. Hubbard, P. King, J. Logan, L. Muench, D. Pareto, et al. Unique distribution of aromatase in the human brain: In vivo studies with pet and [n-methyl-11c] vorozole. Synapse, 64(11):801-807, 2010.

M. A. Boksem, P. H. Mehta, B. Van den Bergh, V. van Son, S. T. Trautmann, K. Roelofs, A. Smidts, and A. G. Sanfey. Testosterone inhibits trust but promotes reciprocity. Psychological science, 24(11):2306-2314, 2013.

A. O. Brinkmann. Molecular mechanisms of androgen action-a historical perspective. Androgen Action, pages 3-24, 2011.

C. F. Camerer. Chapter five. prospect theory in the wild: Evidence from the field. In Advances in behavioral economics, pages 148-161. Princeton University Press, 2011.

Z. Carmon and D. Ariely. Focusing on the forgone: How value can appear so different to buyers and sellers. Journal of consumer research, 27(3):360-370, 2000.

E. Y. Chan. Endowment effect for hedonic but not utilitarian goods. International Journal of Research in Marketing, 32(4):439-441, 2015.

B. R. Cherki, E. Winter, D. Mankuta, and S. Israel. Intranasal oxytocin, testosterone reactivity, and human competitiveness. Psychoneuroendocrinology, 132:105352, 2021.

L. A. Chicaiza-Becerra and M. Garcia-Molina. Prenatal testosterone predicts financial risk taking: Evidence from latin america. Personality and Individual Differences, 116:32-37, 2017.

L. Cramer and G. Antonides. Endowment effects for hedonic and utilitarian food products. Food quality and preference, 22(1):3-10, 2011.

C. Cueva, R. E. Roberts, T. Spencer, N. Rani, M. Tempest, P. N. Tobler, J. Herbert, and A. Rustichini. Cortisol and testosterone increase financial risk taking and may destabilize markets. Scientific reports, 5(1):1-16, 2015

B. Derntl, N. Pintzinger, I. Kryspin-Exner, and V. Schöpf. The impact of sex hormone concentrations on decision-making in females and males. Frontiers in neuroscience, 8:352, 2014.

R. Dhar and K. Wertenbroch. Consumer choice between hedonic and utilitarian goods. Journal of marketing research, 37(1):60-71, 2000.

J.-C. Dreher, P. J. Schmidt, P. Kohn, D. Furman, D. Rubinow, and K. F. Berman. Menstrual cycle phase modulates reward-related neural function in women. Proceedings of the National Academy of Sciences, 104(7):2465-2470, 2007.

K. M. Durante and V. Griskevicius. Evolution and consumer behavior. Current Opinion in Psychology, 10:27-32, 2016.

K. M. Durante, V. Griskevicius, S. M. Cantu, and J. A. Simpson. Money, status, and the ovulatory cycle. Journal of Marketing Research, 51(1):27-39, 2014.

C. Eisenegger, J. Haushofer, and E. Fehr. The role of testosterone in social interaction. Trends in cognitive sciences, 15(6):263-271, 2011

C. R. Fox and R. A. Poldrack. Prospect theory and the brain. In Neuroeconomics, pages 145-173. Elsevier, 2009.

S. Gächter, E. J. Johnson, and A. Herrmann. Individual-level loss aversion in riskless and risky choices. Theory and Decision, pages 1-26, 2021.

E. Garbarino, R. Slonim, and J. Sydnor. Digit ratios (2d: 4d) as predictors of risky decision making for both sexes. Journal of Risk and Uncertainty, 42(1):1-26, 2011.

A. E. Goudriaan, B. Lapauw, J. Ruige, E. Feyen, J.-M. Kaufman, M. Brand, and G. Vingerhoets. The influence of high-normal testosterone levels on risk-taking in healthy males in a 1-week letrozole administration study. Psychoneuroendocrinology, 35(9):1416-1421, 2010.

E. C. Hirschman and M. B. Holbrook. Hedonic consumption: emerging concepts, methods and propositions. Journal of marketing, 46(3):92-101, 1982 
P. Höfer, R. Lanzenberger, and S. Kasper. Testosterone in the brain: neuroimaging findings and the potential role for neuropsychopharmacology. European Neuropsychopharmacology, 23 (2):79-88, 2013.

A. M. Hughes, S. Harrison, L. D. Howe, and N. M. Davies. Testosterone and socioeconomic position: Mendelian randomization in 306,248 men and women participants of uk biobank. Science Advances, 2021.

D. Kahneman and A. Tversky. Prospect theory: An analysis of decision under risk, econometrica, vol. 47, pp 263-291, 1979.

D. Kahneman, J. L. Knetsch, and R. H. Thaler. Anomalies: The endowment effect, loss aversion, and status quo bias. Journal of Economic perspectives, 5(1):193-206, 1991.

J. L. Knetsch. The endowment effect and evidence of nonreversible indifference curves. The american Economic review, 79(5):1277-1284, 1989

F. H. Knight. Risk, uncertainty and profit, volume 31. Houghton Mifflin, 1921.

J. Kurath and R. Mata. Individual differences in risk taking and endogeneous levels of testosterone, estradiol, and cortisol: A systematic literature search and three independent metaanalyses. Neuroscience \& Biobehavioral Reviews, 90:428-446, 2018.

R. McElreath. Statistical rethinking: A Bayesian course with examples in $R$ and Stan. Chapman and Hall/CRC, 2018.

P. H. Mehta, K. M. Welker, S. Zilioli, and J. M. Carré. Testosterone and cortisol jointly modulate risk-taking. Psychoneuroendocrinology, 56:88-99, 2015.

A. Nadler, M. Wibral, T. Dohmen, A. Falk, A. Previtero, B. Weber, C. Camerer, A. Dreber, and G. Nave. Does testosterone administration increase competitiveness, confidence and financial risk-taking in men?, Aug 2021. URL psyarxiv. com/62af7.

G. Nave, A. Nadler, D. Dubois, D. Zava, C. Camerer, and H. Plassmann. Single-dose testosterone administration increases men's preference for status goods. Nature Communications, 9(1): $1-8,2018$.

M. V. Nepomuceno, G. Saad, E. Stenstrom, Z. Mendenhall, and F. Iglesias. Testosterone \& giftgiving: Mating confidence moderates the association between digit ratios (2d: $4 \mathrm{~d}$ and rel2) and erotic gift-giving. Personality and Individual Differences, 91:27-30, 2016a.

M. V. Nepomuceno, G. Saad, E. Stenstrom, Z. Mendenhall, and F. Iglesias. Testosterone at your fingertips: Digit ratios (2d: $4 \mathrm{~d}$ and rel2) as predictors of courtship-related consumption intended to acquire and retain mates. Journal of Consumer Psychology, 26(2):231-244, 2016b.

H. Nilsson, J. Rieskamp, and E.-J. Wagenmakers. Hierarchical bayesian parameter estimation for cumulative prospect theory. Journal of Mathematical Psychology, 55(1):84-93, 2011.

H. Nilsson, J. Rieskamp, and E.-J. Wagenmakers. Hierarchical bayesian parameter estimation for cumulative prospect theory. Journal of Mathematical Psychology, 98:102429, 2020.

A. A. Puiu, S. Radke, M. Votinov, U. Habel, B. Herpertz-Dahlmann, B. Turetsky, and K. Konrad. Serum testosterone and cortisol concentrations after single-dose administration of $100-\mathrm{mg}$ transdermal testosterone in healthy men. Frontiers in pharmacology, 10:1397, 2019.

H. Pushkarskaya, P. Lenkic, B. Stewart, D. Tolin, and S. R. Woody. Hoarding symptoms correlate with the endowment effect. Journal of Behavioral and Cognitive Therapy, 30(3):201-210, 2020.

R. Ronay, L. van der Meij, J. Oostrom, and T. Pollet. No evidence for a relationship between testosterone hair samples and 2d: $4 \mathrm{~d}$ ratio or risk taking. Frontiers in Behavioral Neuroscience, 12, 2018.

K. Ruggeri, S. Alí, M. L. Berge, G. Bertoldo, L. D. Bjørndal, A. Cortijos-Bernabeu, C. Davison, E. Demić, C. Esteban-Serna, M. Friedemann, et al. Replicating patterns of prospect theory for decision under risk. Nature Human Behaviour, pages 1-12, 2020.

G. Saad and J. G. Vongas. The effect of conspicuous consumption on men's testosterone levels. Organizational Behavior and Human Decision Processes, 110(2):80-92, 2009.

P. Sapienza, L. Zingales, and D. Maestripieri. Gender differences in financial risk aversion and career choices are affected by testosterone. Proceedings of the National Academy of Sciences, 106(36):15268-15273, 2009.

R. M. Sapolsky and M. J. Meaney. Maturation of the adrenocortical stress response: neuroendocrine control mechanisms and the stress hyporesponsive period. Brain research reviews, 11(1):65-76, 1986.

H. Sasano, K. Takahashi, F. Satoh, H. Nagura, and N. Harada. Aromatase in the human central nervous system. Clinical endocrinology, 48(3):325-329, 1998.

O. C. Schultheiss and S. J. Stanton. Assessment of salivary hormones. Methods in social neuroscience, 17, 2009

R. M. Shiffrin, M. D. Lee, W. Kim, and E.-J. Wagenmakers. A survey of model evaluation approaches with a tutorial on hierarchical bayesian methods. Cognitive Science, 32(8):1248$1284,2008$.

V. Singh. Role of cortisol and testosterone in risky decision-making: Deciphering male decisionmaking in the iowa gambling task. Frontiers in Neuroscience, 15, 2021.

S. J. Stanton. The role of testosterone and estrogen in consumer behavior and social \& economic decision making: A review. Hormones and behavior, 92:155-163, 2017.

S. J. Stanton, S. H. Liening, and O. C. Schultheiss. Testosterone is positively associated with risk taking in the iowa gambling task. Hormones and behavior, 59(2):252-256, 2011a.

S. J. Stanton, O. A. Mullette-Gillman, R. E. McLaurin, C. M. Kuhn, K. S. LaBar, M. L. Platt, and S. A. Huettel. Low-and high-testosterone individuals exhibit decreased aversion to economic risk. Psychological science, 22(4):447-453, 2011b.

S. J. Stanton, K. M. Welker, P. L. Bonin, B. Goldfarb, and J. M. Carré. The effect of testosterone on economic risk-taking: A multi-study, multi-method investigation. Hormones and Behavior 134:105014, 2021.

M. Strahilevitz and J. G. Myers. Donations to charity as purchase incentives: How well they work may depend on what you are trying to sell. Journal of consumer research, 24(4):434-446, 1998.

J. M. Sundie, D. T. Kenrick, V. Griskevicius, J. M. Tybur, K. D. Vohs, and D. J. Beal. Peacocks, porsches, and thorstein veblen: Conspicuous consumption as a sexual signaling system. Journal of personality and social psychology, 100(4):664, 2011.

D. Terburg, S. Syal, L. A. Rosenberger, S. J. Heany, D. J. Stein, and J. van Honk. Testosterone abolishes implicit subordination in social anxiety. Psychoneuroendocrinology, 72:205-211, 2016.

R. Thaler. Toward a positive theory of consumer choice. Journal of economic behavior \& organi- zation, 1(1):39-60, 1980

A. Tversky and D. Kahneman. Loss aversion in riskless choice: A reference-dependent model. The quarterly journal of economics, 106(4):1039-1061, 1991.

A. Tversky and D. Kahneman. Advances in prospect theory: Cumulative representation of uncertainty. Journal of Risk and uncertainty, 5(4):297-323, 1992.

M. J. Van der Loos, R. Haring, C. A. Rietveld, S. E. Baumeister, P. J. Groenen, A. Hofman, F. H. de Jong, P. D. Koellinger, T. Kohlmann, M. A. Nauck, et al. Serum testosterone levels in males are not associated with entrepreneurial behavior in two independent observational studies. Physiology \& behavior, 119:110-114, 2013.

E. Van Dijk and D. Van Knippenberg. Trading wine: On the endowment effect, loss aversion, and the comparability of consumer goods. Journal of Economic Psychology, 19(4):485-495, 1998.

J. van Honk, D. J. Schutter, E. J. Hermans, P. Putman, A. Tuiten, and H. Koppeschaar. Testosterone shifts the balance between sensitivity for punishment and reward in healthy young women. Psychoneuroendocrinology, 29(7):937-943, 2004.

J. Van Honk, E. R. Montoya, P. A. Bos, M. Van Vugt, and D. Terburg. New evidence on testosterone and cooperation. Nature, 485(7399):E4-E5, 2012.

A. L. Vermeer, M. Boksem, C. Gausterer, C. Eisenegger, and C. Lamm. Testosterone increases risk-taking for status but not for money. PsyArXiv, 2020.

M. Votinov, T. Mima, T. Aso, M. Abe, N. Sawamoto, J. Shinozaki, and H. Fukuyama. The neural correlates of endowment effect without economic transaction. Neuroscience research, 68(1): 59-65, 2010.

M. Votinov, T. Aso, S. Koganemaru, H. Fukuyama, and T. Mima. Transcranial direct current stimulation changes human endowment effect. Neuroscience research, 76(4):251-256, 2013.

M. Votinov, L. Wagels, F. Hoffstaedter, T. Kellermann, K. S. Goerlich, S. B. Eickhoff, and U. Habel. Effects of exogenous testosterone application on network connectivity within emotion regulation systems. Scientific reports, 10(1):1-10, 2020.

L. Wagels, M. Votinov, S. Radke, B. Clemens, C. Montag, S. Jung, and U. Habel. Blunted insula activation reflects increased risk and reward seeking as an interaction of testosterone administration and the maoa polymorphism. Human Brain Mapping, 38(9):4574-4593, 2017.

Y. Wu, J. Liu, L. Qu, C. Eisenegger, L. Clark, and X. Zhou. Single dose testosterone administration reduces loss chasing in healthy females. Psychoneuroendocrinology, 71:54-57, 2016.

Y. Wu, S. Zilioli, C. Eisenegger, L. Clark, and H. Li. The effect of testosterone administration and digit ratio (2d: $4 \mathrm{~d}$ ) on implicit preference for status goods in healthy males. Frontiers in Behavioral Neuroscience, 11:193, 2017.

N. Zethraeus, L. Kocoska-Maras, T. Ellingsen, B. Von Schoultz, A. L. Hirschberg, and M. Johannesson. A randomized trial of the effect of estrogen and testosterone on economic behavior. Proceedings of the National Academy of Sciences, 106(16):6535-6538, 2009. 


\section{Supplementary Note 1: Bayesian models estimation details}

Below we provided specification for Bayesian model used in paper. Despite the power of hierarchical models, there are a lot of subtle problems appearing during the estimation of these models, and to overcome convergence problems we used a so-called "reparameterization trick", specifically non-centered parametrization McElreath (2018). The only difference is in the way of model formulation, in case of Normal distribution with parameters $\mu$ and $\sigma$ we can sample directly from $\operatorname{Normal}(\mu, \sigma)$ or from $\mu+\sigma *(\operatorname{Normal}(0,1))$. The outcome is absolutely identical, but sampling procedure differs. So, we used non-centered priors in all the models. Also, for all the models posterior distributions were approximated by a total of 24,000 MCMC samples obtained from four chains, after a burn-in of 1000 samples. Convergence of the MCMC chains was confirmed by visual inspection and by computing relevant $\mathrm{R}$ hat statistics.

\section{Endowment effect model specification.}

The WTA/WTP ratio measures endowment effect. In this paradigm, willingness to pay (WTP) to acquire an object is typically lower than the least amount they are willing to accept (WTA) to give up that same object when they own it-even when there is no cause for attachment. The hypothesis to test:

1. WTA/WTP $>1$ There is endowment effect for both type of goods (utilitarian and hedonic)

2. WTA/WTP ${ }_{h}>\mathrm{WTA} \mathrm{WTP}_{u}$ Endowment effect for hedonic goods bigger then for utilitarian

3. WTA/WTP $T$ T $>$ WTA/WTP $P$ Testosterone administration will increase endowment effect, especially for hedonic goods.

For these hypothesis testing we estimated two models. The first one for endowment effect existence for two type of goods regardless individual and item differences and without testosterone-related parameters included and the second one with individual and item differences and with testosterone-related shift parameter.

\section{Model 1.}

Bayesian model: WTA/WTP ratio came from lognormal distribution with unknown mean and variance. Model specification:

* Likelihood: WTA/WTP $\sim \operatorname{LogNormal}\left(\mu_{\text {Type }}, \sigma_{\text {Type }}\right)$

* Priors: informative prior for checking H0 hypothesis: mean $=0$, what corresponds 1 (equal WTA and WTP) in real scale, because in logarithmic because $\exp (0)=1$. Formally:

- $\mu \sim \operatorname{Normal}($ mean $=0, \sigma=1$, size $=2$ ), mean prior

- $\sigma \sim \operatorname{Exponential}(1$, size $=2)$, uniformative prior for sigma

Lognormal distribution for WTA/WTP ratio was chosen because ratio is strongly positive and skewed as evidenced by empirical distributions as could be seen at the Figure 11

For assessing the plausibility of the model we draw posterior predictive distribution for the WTA/WTP ratio based on the fitted Bayesian model. We can see from the Figure 12 that observed data set is consistent with the model.
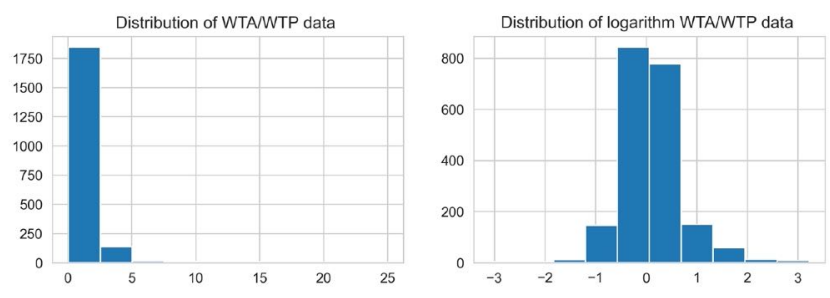

Fig. 11. Histogram for raw WTA/WTP data and their logarithm

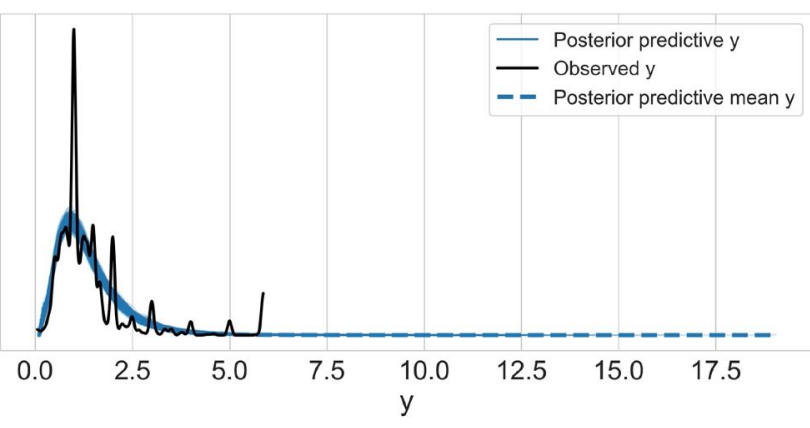

Fig. 12. Posterior predictive distribution for Model 1

\section{Model 2.}

In this model we used the same we used hierarchical approach and added item differences and individual differences at the second level and item differences and with testosterone-related shift parameter Tchange computed as relative difference in testosterone level for placebo and hormone condition for each participant.

* Likelihood: $\log \left(\mathrm{WTA} / \mathrm{WTP}_{[i, j]}\right)=\mu_{0}+\beta *$ Tchange $+\mu_{\mathrm{ind}[j]}+g_{\mathrm{g}[i]}$ 
* Priors:

- $\mu_{0} \sim \operatorname{Normal}(0,0.5)$ (group mean)

- $\sigma \sim \operatorname{Exponential(1)~(model~error)~}$

- $\sigma_{g}=\operatorname{Exponential}(2)$ (between item deviation)

- $\sigma_{\text {ind }}=\operatorname{Exponential}(2)$ (between subject deviation)

- $\beta \sim \operatorname{Normal}(0,0.5$, size $=$ Nitems) (sensitivity to testosterone level change, different for each item)

- $z_{\mathrm{g}} \sim \operatorname{Normal}\left(0,1\right.$, size $=$ Number of items), $g_{\mathrm{g}}=z_{\mathrm{g}} * \sigma_{\mathrm{g}}$ (item mean shift)

- $z_{\text {ind }} \sim \operatorname{Normal}(0,1$, size $=$ Number of subjects $), \mu_{\text {ind }}=z_{\text {ind }} * \sigma_{\text {ind }}$ (subject mean shift)

\section{Replication study.}

Open dataset explored in Nilsson et al. (2011) includes 30 participants and 180 games, each game is a chance prospect with two non-zero outcomes $x, p ; y,(1-p)$, data available at https://osf.io/sbxm2/. In those games there were 60 games with pure gain prospects, 60 with pure loss and 60 with mixed prospects. Apart from our study both options $A, B$ are chance prospects, and there is no reason to prefer one of them by default, so we excluded intercept parameter. We replicated the hierarchical model suggested in the original paper, but only for pure gain and pure loss (positive and negative framing) games, because we did not have mixed games in our study. We also estimated the model with log transform modification. From prospect theory (see section Prospect Theory) in case of two games with different non-zero probabilities:

$$
V(A)=\sum v\left(x_{i}\right) * \omega\left(p_{i}\right)
$$

where $V(A)$ - value function, $\omega\left(p_{i}\right)$ - decision weights for for gains and losses

$$
\begin{gathered}
v\left(x_{i}\right)= \begin{cases}x^{\alpha_{\text {cond }},} & \text { if }(x)>0 \\
-\lambda(-x)^{\alpha_{\text {cond }}}, & \text { else }\end{cases} \\
\omega(p)=\frac{p^{c_{\text {cond }}}}{\left(p^{c_{\text {cond }}}+(1-p)^{c_{\text {cond }}}\right)^{1} / c_{\text {cond }}}
\end{gathered}
$$

where cond $=+$, if positive, - else is a game type

Choice function, probability of choosing option B over A:

$$
\theta=\frac{1}{1+e^{-\phi(V(B)-V(A))}}
$$

We slightly modify the equations for computational purpose. We considered only pure loss and pure win games, so we can use different $\phi$ for each treatment instead of $\lambda$ :

$$
V(B)-V(A)=\lambda *(\ldots)
$$

so we can use $\phi_{\text {cond }}$, where $\phi_{-}=\lambda * \phi_{+}$

\section{Model specification.}

* Likelihood:

- $y_{i} \sim \operatorname{Bernoulli}\left(\theta_{i}\right)$ (binary outcome)

- Choice probability for game with two outcomes $(\mathrm{x}, \mathrm{y})$, with the second outcome probability equal to q modeled with logistic regression.

$$
\left\{\begin{array}{l}
\operatorname{logit}\left(\theta_{i}\right)=\phi_{i n d} * \operatorname{gap}\left(A, B, \alpha_{i n d}, c_{i n d}\right) \\
\operatorname{gap}(A, B)=\operatorname{Val}\left(B, \alpha_{\text {ind }}, c_{\text {ind }}\right)-\operatorname{Val}\left(A, \alpha_{\text {ind }}, c_{\text {ind }}\right), \text { original model } \\
\operatorname{gap}(A, B)=\operatorname{sign}(\operatorname{gap}(A, B)) * \log (1+\operatorname{abs}(\operatorname{gap}(A, B))), \text { modification used in this paper } \\
\operatorname{Val}(\operatorname{Game}=x, 1-q, y, q)=\left(1-w_{q}\left(c_{\text {cond }}\right)\right) v\left(x, \alpha_{\text {cond }}\right)+w_{q}\left(c_{\text {cond }}\right) v\left(y, \alpha_{\text {cond }}\right)
\end{array}\right.
$$

* Priors. All the model parameters have constraint boundaries, $\alpha$ and $c$ can be in the range $[0,1] \phi$ could take only positive values. So, we couldn't take the normal distribution for priors. However, on the probit scale the parameters cover the entire real line, so additional normally distributed variables were introduces and transformed after that with probit scale to [0-1] range and exponentially to positive range. Individual differences introduces with hierarchical model, but unlike original paper we used non-centered parametrisation. Precisely, the individual parameters come from independent group-level normal distributions 
- Priors for $\alpha$ parameter which modulate the curvature of the subjective value functions, group level and individual:

$$
\left\{\begin{array}{l}
\alpha N_{\text {cond }} \sim \operatorname{Normal}(0,5, \text { size }=2), \\
\alpha_{\text {cond }} \sim \operatorname{probit}\left(\alpha N_{\text {cond }}\right)
\end{array}\right.
$$

- Priors for $\mathrm{c}$ specifies the transformation of the weighting function, group level and individual:

$$
\left\{\begin{array}{l}
c N_{\text {cond }} \sim \operatorname{Normal}(0,5, \text { size }=2) \\
c_{\text {cond }} \sim \operatorname{probit}\left(c N_{\text {cond }}\right)
\end{array}\right.
$$

- Priors for sensitivity parameter $\phi$ :

$$
\left\{\begin{array}{l}
\phi N_{\text {cond }} \sim \operatorname{Normal}(0,5, \text { size }=2) \\
\phi_{\text {cond }} \sim \exp \left(\phi N_{\text {cond }}\right)
\end{array}\right.
$$

- Non-centered priors for individual differences in parameters:

$$
\left\{\begin{array}{l}
\alpha_{i n d}=\operatorname{probit}\left(\alpha N_{c o n d}+z_{\alpha_{i n d}} * \sigma_{\alpha_{\text {ind }}}\right) \\
c_{\text {ind }}=\operatorname{probit}\left(c N_{\text {cond }}+z_{c_{\text {ind }}} * \sigma_{c_{\text {ind }}}\right) \\
\phi_{\text {ind }}=\exp \left(\phi N_{\text {cond }}+z_{\phi_{\text {ind }}} * \sigma_{\phi_{\text {ind }}}\right)
\end{array}\right.
$$

\section{Risk-taking model.}

The measured data from the experiment is presented as binary choice outcomes, so the data generation process is a binomial distribution.

* Likelihood:

- $y_{i} \sim \operatorname{Bernoulli}\left(\theta_{i}\right)$, with $\theta$ probability of taking risk in each game

- The probability $\theta$ is modelled with logistic regression

$$
\operatorname{logit}(\theta)=\operatorname{Intercept}+\operatorname{Beta} * \operatorname{gap}(A, B, \alpha, c)
$$

- For parameters A and B, there is a variety of options for modelling. Since both slope and intercept are considered a function of framing $(\mathrm{F})$ and testosterone application, we added it as a linear term multiplied by the rate of change in testosterone level relative to placebo denoted as Tchange below.

$$
\left\{\begin{array}{l}
\text { Intercept }=a_{F}+\delta_{-} a_{F} * \text { Tchange } \\
\text { Beta }=b_{F}+\delta_{-} b_{F} * \text { Tchange }
\end{array}\right.
$$

* Priors specification In our model, we suppose that there is a framing-dependent group-level $a_{F}$ sampled from a normal distribution. Individual parameters $a_{\text {ind }}$ sampled from a normal distribution with group-level mean and standard deviation. The same stands true for $B$, which is the sensitivity to payout difference. However, it is unreasonable to suggest negative sensitivity to the gap, so we also choose normal prior but with 0.5 mean and standard deviation equal to 0.5 . For testosterone-related terms $\delta_{\_} a_{F}$ and $\delta_{-} b_{F}$ we did not use a hierarchical approach, because individual differences mostly were taken into account through independent variable Tchange - change in testosterone level. For them, it is also natural to choose normal priors with zero mean and unit variance. As a result, we have four risk-taking related group-level parameters, and four testosterone risk-taking related. Since it is natural for an individual to suggest interdependence from all parameters, we suppose that a risk-seeking subject will demonstrate it in both framings or there will at least be some correlation, both for intercept and slope parameters. Also, we take into account the covariation between intercepts and slopes in each framing. Formally,

$$
A \sim \operatorname{MvNormal}\left(\left[a_{F}+\delta_{-} a_{F}, b_{F}+\delta_{-} b_{F}\right], \Sigma\right)
$$

Figure with the model description 13: 


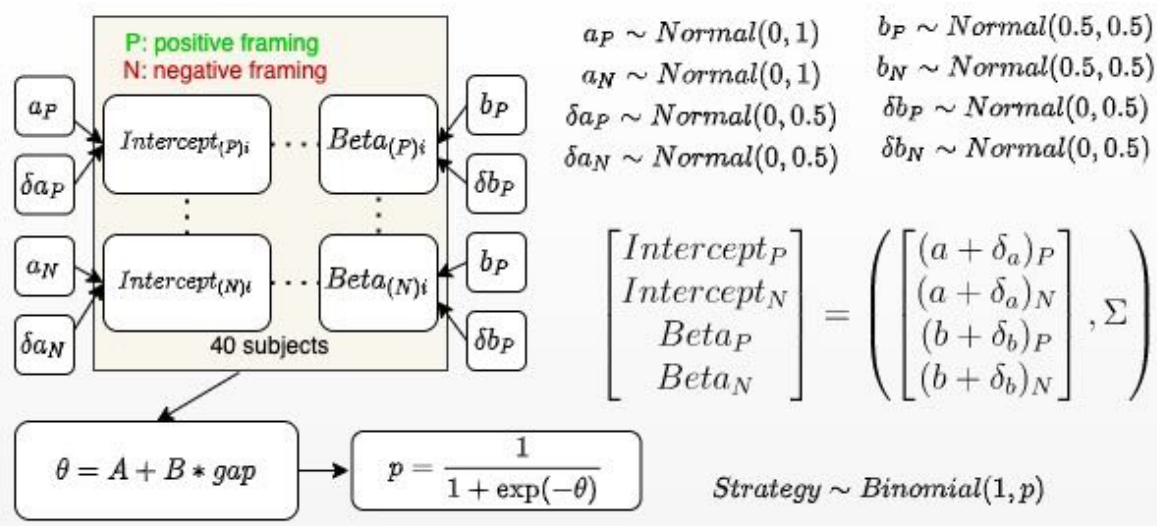

Fig. 13. Prior specification for risk-taking model 